\title{
Quantum Prisoner's Dilemma game on hypergraph networks
}

\author{
Eukasz Pawela* \\ Institute of Theoretical and Applied Informatics, \\ Polish Academy of Sciences, Battycka 5, 44-100 Gliwice, Poland \\ Jan Sładkowsk $\dagger^{\dagger}$ \\ Institute of Physics, University of Silesia, Uniwersytecka 4, 40-007 Katowice, Poland
}

(Dated: 27 February 2012)

\begin{abstract}
We study the possible advantages of adopting of quantum strategies in multi-player evolutionary games. We base our study on the three-player Prisoner's Dilemma (PD) game. In order to model the simultaneous interaction between three agents we use hypergraphs and hypergraph networks. In particular, we study two types of networks: a random network and a SF-like network. The obtained results show that in the case of a three player game on a hypergraph network, quantum strategies are not necessarily stochastically stable strategies. In some cases, the defection strategy can be as good as a quantum one.
\end{abstract}

PACS numbers: 03.67.-a, 02.50.Le

\section{INTRODUCTION}

Game theory is a branch of mathematics broadly applied in a great number of fields, from biology to social sciences and economics. A great deal of effort has gone into the study of evolutionary games on graphs, which was initiated by the work of Nowak and May 11. Since their work was published, a lot of effort was put into studying the problem [2].

Quantum game theory [3] allows the agents to use quantum strategies. The set of quantum strategies is much larger than a classical one; hence it offers possibility for much more diverse behavior of agents in the network. It has been shown that if only one player is aware of the quantum nature of the system, he/she will never lose in some types of games 4. Recently, it has been demonstrated that a player can cheat by appending additional qubits to the quantum system [5].

Combining evolutionary games and quantum game theory, has resulted in absorbing results 6]. In some cases the quantum strategies can dominate the entire network, infecting it effectively. In our work we like to focus on introducing additional strategies which the agents can use, since in the multi-player case there exists a Pareto Optimal Nash Equilibrium for the Prisoner's Dilemma game 7]. Moreover, the PD game is interesting to study, because it was realized experimentally [8].

A hypergraph is a concept that generalizes the concept of a graph by allowing edges to connect more than two nodes at once. This concept can be applied for systems with evolution described by the extended spin-1/2 chain 9. Based on this, the notion of hypergraphs was proven to be a useful tool for assuring controllability of multipartite quantum systems 10 .

\footnotetext{
* lukasz.pawela@gmail.com

$\dagger$ jan.sladkowski@us.edu.pl
}

This paper is organized as follows: In Section [I] a short review of quantum game theory is given and in Section III the types of 3-hypergraph networks used in simulations are described. Section IV introduces the three-player Prisoner's Dilemma game. In Section V the simulation setup is described. Section VI contains results obtained from computer simulations and their discussion. Finally, in Section VII the final conclusions are drawn.

\section{QUANTUM GAME THEORY}

Games that admit the player to use the peculiarities of quantum phenomena are referred to as quantum games [3, 4, 11. Of course, they are games in the "classical" sense. Actually, any quantum system which can be manipulated by at least one party and where the utility of the moves can be reasonably quantified, may be conceived as a quantum game. To be more specific, a twoplayer quantum game $\Gamma=\left(\mathcal{H}, \rho, S_{A}, S_{B}, P_{A}, P_{B}\right)$ is completely specified by the underlying Hilbert space $\mathcal{H}$ of the physical system, the initial state $\rho \in \mathcal{S}(\mathcal{H})$, where $\mathcal{S}(\mathcal{H})$ is the associated state space, the sets $S_{A}$ and $S_{B}$ of permissible quantum operations of the two players, and the pay-off (utility) functions $P_{A}$ and $P_{B}$, which specify the pay-off for each player. A quantum strategy $s_{A} \in S_{A}$, $s_{B} \in S_{B}$ is a quantum operation, that is, a completely positive trace-preserving map mapping the state space on itself. The quantum game's definition may also include certain additional rules, such as the order of the implementation of the respective quantum strategies. The generalization to the multi-player case is straightforward. Schematically we have:

$$
\rho \mapsto\left(s_{A}, s_{B}\right) \mapsto \sigma \Rightarrow\left(P_{A}, P_{B}\right)
$$

The following concepts are commonly used in the context of quantum game theory. These definitions are fully analogous to the corresponding definitions in "classical" 
game theory. A quantum strategy $s_{A}$ is called dominant strategy of Alice if

$$
P_{A}\left(s_{A}, s_{B}^{\prime}\right) \geq P_{A}\left(s_{A}^{\prime}, s_{B}^{\prime}\right)
$$

for all $s_{A}^{\prime} \in S_{A}, s_{B}^{\prime} \in S_{B}$. Analogously we can define a dominant strategy for Bob. A pair $\left(s_{A}, s_{B}\right)$ is said to be an equilibrium in dominant strategies if $s_{A}$ and $s_{B}$ are the players' respective dominant strategies. A combination of strategies $\left(s_{A}, s_{B}\right)$ is called a Nash equilibrium if

$$
\begin{aligned}
& P_{A}\left(s_{A}, s_{B}\right) \geq P_{A}\left(s_{A}^{\prime}, s_{B}\right), \\
& P_{B}\left(s_{A}, s_{B}\right) \geq P_{B}\left(s_{A}, s_{B}^{\prime}\right) .
\end{aligned}
$$

A pair of strategies $\left(s_{A}, s_{B}\right)$ is called Pareto optimal, if it is not possible to increase one player's pay-off without lessening the pay-off of the other player. A solution in dominant strategies is the strongest solution concept for a non-zero sum game. In the Prisoner's Dilemma [3, 4, :

\begin{tabular}{l|cc} 
& Bob $: C$ & Bob $: D$ \\
\hline Alice $: C$ & $(3,3)$ & $(0,5)$ \\
Alice $: D$ & $(5,0)$ & $(1,1)$
\end{tabular}

(the numbers in parentheses represent the row (Alice) and column (Bob) player's payoffs, respectively). Defection is the dominant strategy, as it is favorable regardless what strategy the other party chooses. In a Nash equilibrium neither player has a motivation to unilaterally alter his/her strategy, as this action will not imcrease his/her pay-off. Given that the other player will stick to the strategy corresponding to the equilibrium, the best result is achieved by also playing the equilibrium solution. The concept of Nash equilibrium is therefore of paramount importance. However, it is only an acceptable solution concept if the Nash equilibrium is not unique. For games with multiple equilibria we have to find a way to eliminate all but one of the Nash equilibria. A Nash equilibrium is not necessarily efficient. We say that an equilibrium is Pareto optimal if there is no other outcome which would make all players better off. Up to now a lot of papers on quantum games have been published and some application outside the field of physics have also been discussed [12 18.

\section{HYPERGRAPHS AND HYPERGRAPH NETWORKS}

We assume a hypergraph [19] network $H(X, E)$ where $X$ is a set of nodes and $E$ is a set of non-empty subsets of $X, E \subseteq 2^{X}$. Elements of $E$ are the hyperedges of $H$. We keep within the boundaries of the case when every subset of $X, A \in E$ satisfies $|A|=3$, i.e. every edge of the hypergraph connects three nodes exactly. Hereafter we will refer to this structure as a 3-hypergraph. We set $N=|X|-$ the total number of agents.

We construct two types of networks: a random network, in which all hyperedges connect random nodes and a SF-like 20] network. We set the number of hyperedges in the random case to $|E|=10000$. The SF-like network is constructed in the following way: First, a network of $m_{0} \ll N$ all connected nodes is created. Then a new node with $m<m_{0}$ links is added to the network. For each of the $m$ links, a pair of unique nodes is chosen from the existing network and a new hyperedge is added. The probability of a node $i$ being chosen is given by:

$$
p_{s f}(i)=\frac{k_{i}}{\sum_{j \in X} k_{j}},
$$

where $k$ is the degree of a node. This procedure is repeated until the number of nodes of the network reaches N.

\section{THREE-PLAYER PD GAME}

The classical Prisoner's Dilemma game is as follows: two players can either cooperate $(C)$ or defect $(D)$. When they both cooperate, each receives a payoff of 3 . On the other hand, when they both defect, each receives a payoff of 1 . When one defects, he/she receives a payoff of 5 , while the other gets 0 .

This approach can be extended to a greater number of players. In the three-player case, the payoff matrix is shown in Table I. We can see that every player is bet-

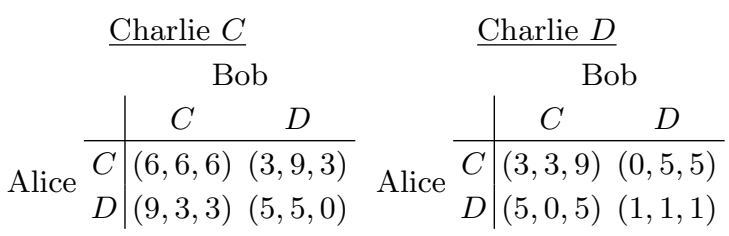

TABLE I: The payoff matrix of the three-player PD game (after [21]). The first entry is the payoff of Alice, the second denotes the payoff of Bob and the third represents the payoff of Charlie.

ter off defecting than cooperating no matter what the other players do. In terms of game theory, $(D, D, D)$ is the unique Nash equilibrium of the game. If any one player deviates from this strategy, he will receive a lower payoff. On the other, we can see that the strategy profile $(C, C, C)$ can yield a higher payoff than $(D, D, D)$. In terms of game theory this profile is Pareto Optimal. In our case the players are rational and the game will end in $(D, D, D)$, not $(C, C, C)$; hence the dilemma.

In the quantum case the setup is as follows. Each player is sent a qubit and can locally operate on it, using any unitary operator $U \in S U(2)$. The initial state of the system is entangled:

$$
|\psi\rangle=J|000\rangle,
$$

where $J$ is the entangling operator [22]:

$$
J=\frac{1}{\sqrt{2}}\left(\mathbb{1}^{\otimes N}+i \sigma_{x}^{\otimes N}\right) .
$$


The quantum circuit for the game is shown in Figure 1. After the players have applied their respective strategies,

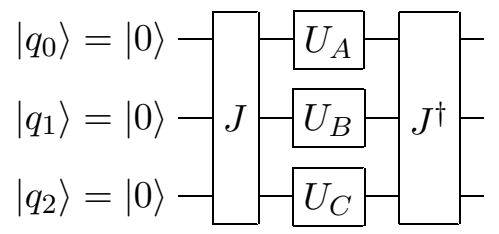

FIG. 1: Quantum circuit for the three-player PD game. $U_{A}, U_{B}, U_{C}$ are the strategies of Alice, Bob and Charlie respectively.

the untangling gate, $J^{\dagger}$, is applied to the system, hence the final state of the game is

$$
\left|\psi_{f}\right\rangle=J^{\dagger}\left(U_{A} \otimes U_{B} \otimes U_{C}\right) J|000\rangle,
$$

where $U_{A}, U_{B}, U_{C}$ are the players strategies. The payoff of the first player (Alice) amounts to:

$$
\$_{A}=\sum_{i, j, k \in\{0,1\}} p_{i j k}\left|\left\langle\psi_{f} \mid i j k\right\rangle\right|^{2},
$$

where $p_{i j k}$ are numbers corresponding to the possible classical payoffs of Alice, defined in Table 1 .

\section{SIMULATIONS}

We assume an initial population of 2500 agents, located at the nodes of a hypergraph. The SF-like network is constructed with initial size $m_{0}=3$, and the number of links of each new node is $m=2$. Throughout all simulations the network topology remains static. The set of allowed strategies is as follows [6]:

$$
S=\{C, D, H, Q\},
$$

where the unitary operators corresponding to each of the strategies take the form of::

$$
\begin{array}{ll}
C=\left(\begin{array}{ll}
1 & 0 \\
0 & 1
\end{array}\right), & D=\left(\begin{array}{ll}
0 & 1 \\
1 & 0
\end{array}\right), \\
H=\frac{1}{\sqrt{2}}\left(\begin{array}{cc}
1 & 1 \\
1 & -1
\end{array}\right), Q=\left(\begin{array}{cc}
i & 0 \\
0 & -i
\end{array}\right) .
\end{array}
$$

In the two-player case, the strategy profile $(Q, Q)$ is a Nash Equilibrium of the game and the strategy $H$ introduces a miracle move when an agent uses it against other's classical strategy [4]. These strategies are randomly assigned to agents in the network in such a way that the initial fractions of strategies $C, D, H, Q$ are $49 \%, 49 \%, 1 \%, 1 \%$ respectively.

Next, we introduce an additional strategy $\Sigma$, defined as:

$$
\Sigma=i \sigma_{y}=\left(\begin{array}{cc}
0 & 1 \\
-1 & 0
\end{array}\right)
$$

The strategy profile $(\Sigma, \Sigma, \Sigma)$ is Pareto Optimal and a Nash Equilibrium [7. We assign the strategies $C, D, H$, $Q, \Sigma$ with frequencies $48 \%, 48 \%, 2 \%, 1 \%, 1 \%$.

Finally, we do not assign strategies randomly, but choose to allocate the strategy $Q$ in the first case and $\Sigma$ in the second one to nodes with the highest degree.

The evolution of strategies of agents is as follows. Initially, each agent is assigned a strategy based on the rules described in the former two paragraphs. Next, agent $i$ plays an entangled quantum game with all other agents forming a hyperedge with him/her. This is repeated for all hyperedges $e \in E$ such that $i \in e$. The total payoff of agent $i, F_{i}$ is obtained by accumulating all the payoffs from these games. After that, the agent $i$ chooses one of these agents randomly, denoted $j$, and imitate its strategy with probability $p_{i}[23$,

$$
p_{i}=\left\{\begin{array}{cc}
\frac{F_{j}-F_{i}}{\alpha \max \left(k_{i}, k_{j}\right)}, & F_{j}>F_{i} \\
0, & \text { otherwise }
\end{array},\right.
$$

where $\alpha=\max \left(\left\{p_{i j k}\right\}\right)$.

The PD game is played by all agents on both networks. We study the impact of the value of the parameter $T$ (moral hazard) on the final state of the population. This parameter is defined as the first players payoff when other players use the $C$ strategy. Its interpretation is as follows. Suppose the prisoners had a chance to discuss a strategy. It is evident that they should decide for a Pareto Optimal profile $(C, C, C)$. However, if Alice decides to defect, she receives a higher payoff. Thus this parameter measures, how much Alice is tempted to betray the other prisoners.

The game is played for 10000 generations and the last 1000 results are stored. Average frequencies of strategies are used as the final results. If a population does not change for 500 generations, the state is considered to be an equilibrium state of the system.

\section{RESULTS AND DISCUSSION}

In all cases, we show results for $\mathrm{T}$ starting from 5 , despite the fact that the game becomes a Prisoner's Dilemma when $T>6$. We have done this to show the behavior of the fractions of strategies near the transition to the PD game.

In the case with four possible strategies, the results of computer simulations are depicted in Figure 2. Figure $2 \mathrm{a}$ shows the results for a random network, whereas the results for the SF-like network are shown in Figure 2b. In the case of a random network, we see that strategy $C$ is the dominant one, until $T=5.64$, when the network starts shifting between strategies $C$ and $D$. It settles down at $T=6$, where about half the agents use strategy $C$. As $T$ increases, strategies $C$ and $D$ slowly lose their significance in favour of strategy $Q$. For $T>8$ the system reaches another equlibrium state, where strategies $D$ and $Q$ have the same frequency. 


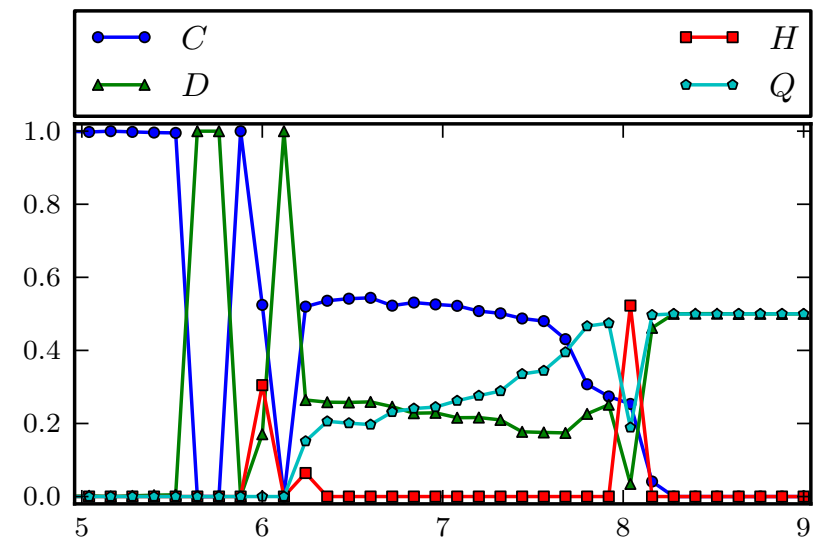

(a) Random network

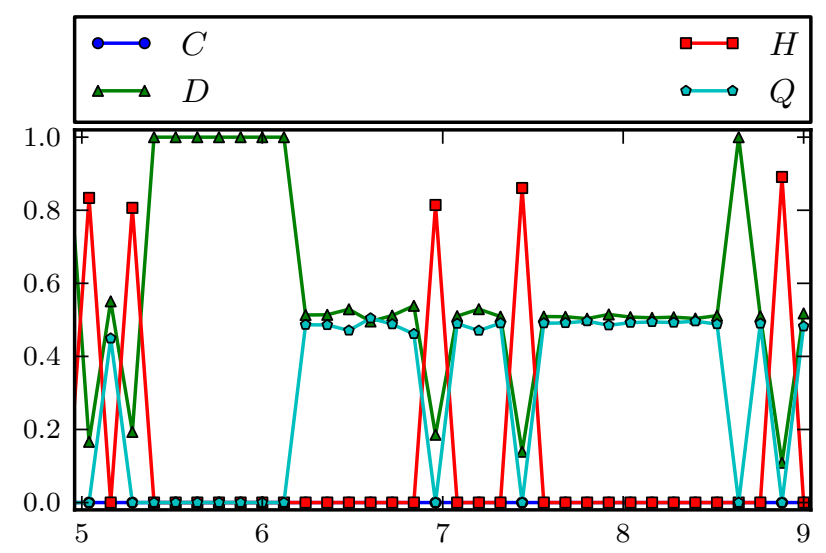

(b) SF-like network

FIG. 2: Results for PD on hypergraph networks, 4 strategies, strategies assigned at random, according to weights.

In the case of a SF-like network, the agents prefer the $D$ strategy, which almost never reaches zero frequency. Again, for $T>6$ we have an increase of significance of the quantum strategy $Q$. Although there are some oscillations of the fraction of strategies as $T$ increases, again strategies $D$ and $Q$ have been adopted by approximately the same fraction of agents. On the basis of the presented figures as well as above discussion it may be inferred that the change of type of the network significantly decreases the importance of strategy $C$, but does not have a great impact on strategies $D$ and $Q$.

Figure 3 illustrates the results obtained for five possible strategies. Figure 3a illustrates the results for a random network, and Figure $3 \mathrm{~b}$ shows the results for the SFlike network. The examination of Figure 3a reveals that it has the same character as the Figure 2a, except that for $T<6$ the dominant strategy is $\Sigma$ not $C$. At around $T=6$, the network shifts from $\Sigma$ dominated to a network with three possible strategies: $C, D, Q$. As $T$ increases, strategies $C$ and $D$ lose their significance in favor of $Q$. At around $T=8$, there is another shift in strategies, and the fraction of strategy $C$ decreases to zero, and strategies $D$ and $Q$ are used by equal fraction of agents.

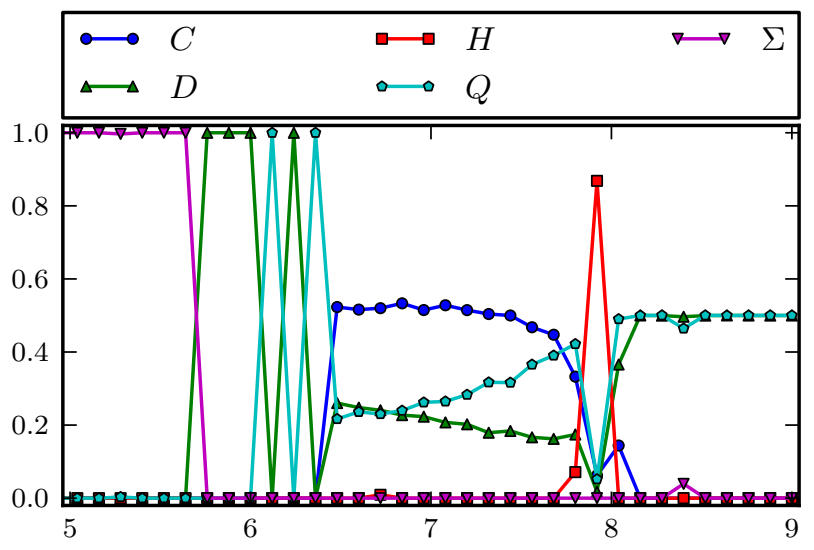

(a) Random network

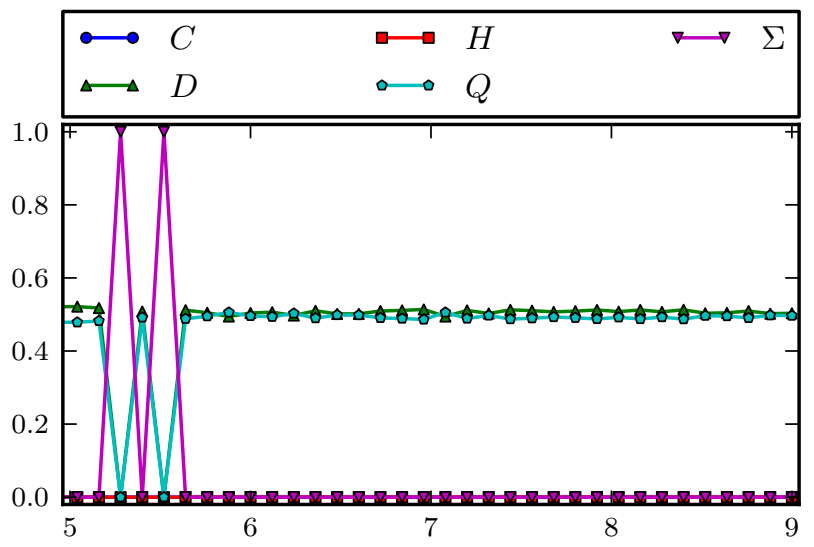

(b) SF-like network

FIG. 3: Results for PD on hypergraph networks, 5 strategies, strategies assigned at random, according to weights.

On examining the SF-like network, we again perceive behaviour similar to the four strategy case, but with much less oscillations. Again for $T>6$ it is observed that strategies $D$ and $Q$ are used by approximately the same number of agents. From the above discussion we observe that the introduction of strategy $\Sigma$ into the network results in two observations. Firstly, for a random network it is the dominant strategy for low Temptations. Secondly, for a SF-like network, it stops some oscillations of the network.

Next we move on to the case, where only one agent, with the highest degree was assigned a quantum strategy. For the case of four available strategies, results are shown in Figure 4. Figure 4a shows the results for a random network and Figure $4 \mathrm{~b}$ shows the results for a SF-like network. In this case the agent with the highest degree was assigned the $Q$ strategy, all other strategies were distributed to the agents with equal probabilities. We perceive, that for $T<6$ the strategy $C$ dominates the network. Again, at around $T=6$ there is a shift, but 
this time the strategy $H$ increases its significance. The fraction of agents using strategy $H$ slowly increases with $T$ increasing. In the case of a SF-like network, we obtain

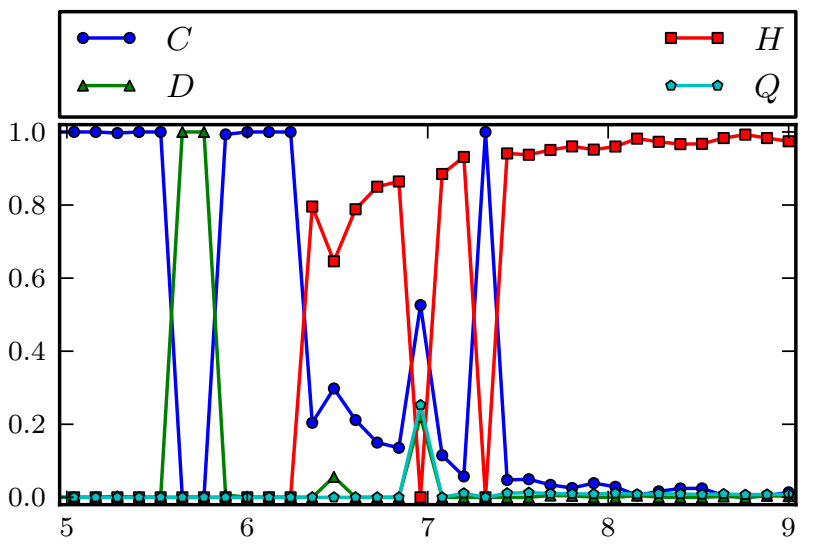

(a) Random network

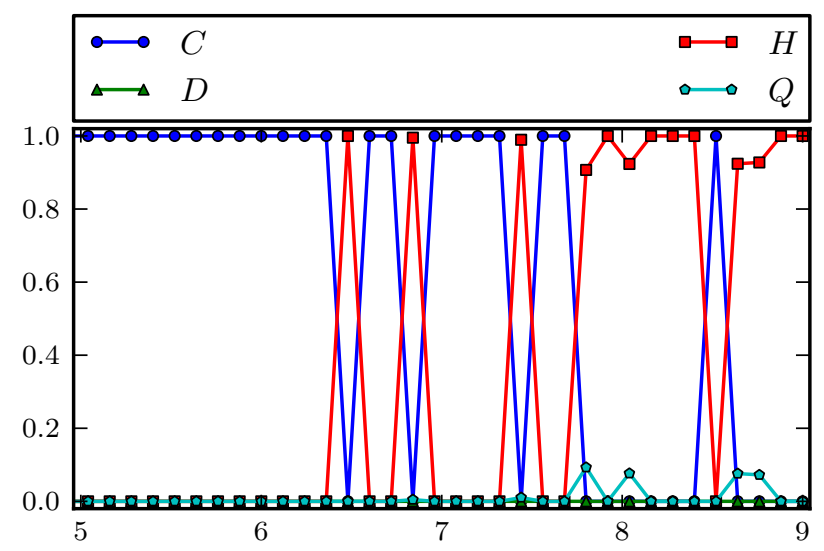

(b) SF-like network

FIG. 4: Results for PD on hypergraph networks, 4 strategies, strategy $Q$ assigned to the node with highest degree.

that for $T<6.5$ the strategy $C$ dominates the network. For greater $T$ the network starts shifting from strategy $C$ to $H$, still a small fraction of agents also use the $Q$ strategy. Summing up this case, we can conclude that strategy $Q$ cannot infect any of the networks, but assigning the strategy $H$ to a relatively big fraction of agents allows it to dominate the network for some values of $T$.

Finally, we show the results for the case with five possible strategies. Now we assign the strategy $\Sigma$ to the agent with the highest degree. The results obtained in this case are shown in Figure 5 . Figure 5a illustrates the results for a random network and Figure $5 \mathrm{~b}$ shows the results for a SF-like network. For a random network, as can be seen still that for $T<6$ the $C$ strategy is employed by all of the agents. As $T$ increases from 6 to 8 , the strategies $C, Q$ and $D$ are used by a significant fraction of agents. At around $T=8$ the strategy $H$ dominates the network. Then, just before $T$ reaches 9 , there is another sudden shift and strategies $D$ and $Q$ are used by the same fraction of agents, with other strategies being far less significant. In the case of SF-like network, we

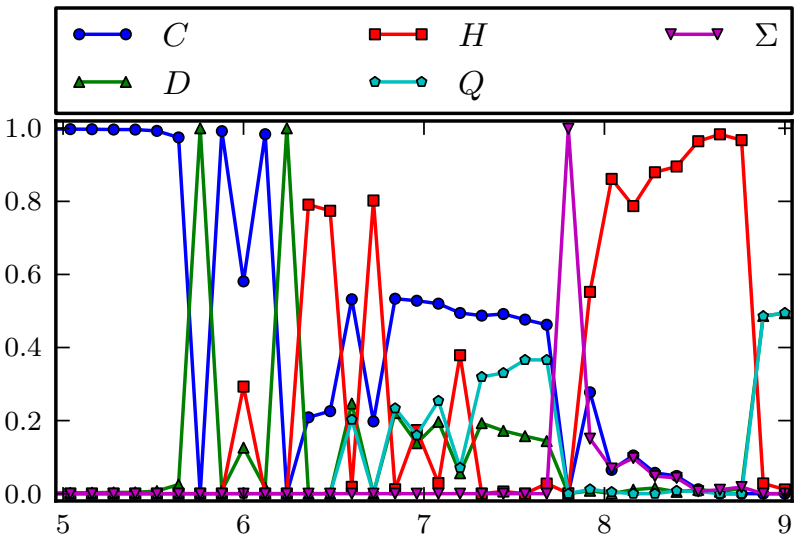

(a) Random network

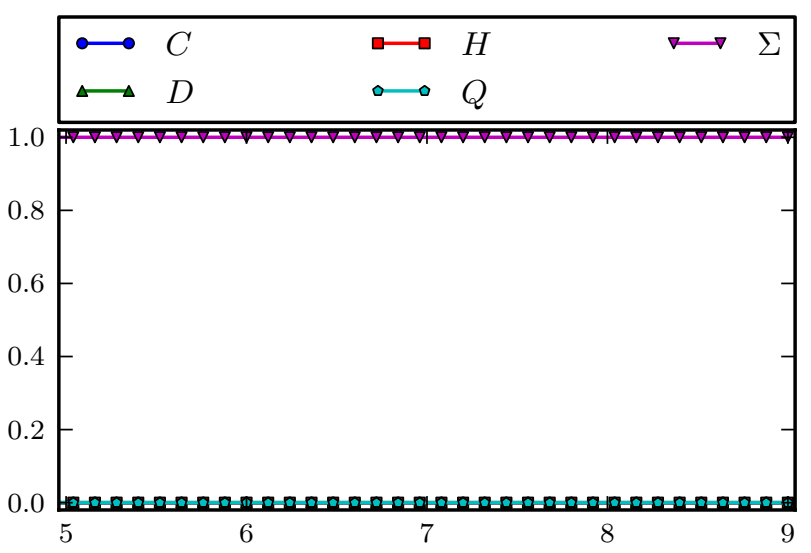

(b) SF-like network

FIG. 5: Results for PD on hypergraph networks, 5 strategies, strategy $\Sigma$ assigned to the node with highest degree.

observe an entirely different behaviour. The strategy $\Sigma$ always dominates the network, regardless of the value of $T$. From this discussion it is evident that the $\Sigma$ strategy can only invade a network of a specific type. A random network is immune to invasion.

\section{CONCLUSIONS}

We investigate the evolution of strategies on hypergraph networks when quantum strategies $H, Q$ and $\Sigma$ are available to the players. Strategies $Q$ and $\Sigma$ are considered to be invaders in our scenario. Our simulations of the evolution of strategies on a random and SF-like hypergraph network indicate that the structure of the network is a decisive factor. In addition, we discovered that, the strategy $\Sigma$, despite being Pareto Optimal and a Nash Equilibrium for the three-player Prisoner's Dilemma game, does not invade the entire network in all cases. In fact, it can only invade a SF-like network, pro- 
vided that the agent with the highest degree is assigned this strategy. In other cases, depending on the value of Temptation, the network is dominated by strategy $C$, what happens for $T<6$, or strategies $D$ and $Q$ have equal frequencies what happens for $T>8$. The results obtained for the case with four available strategies, are slightly different. In this case the strategy $Q$ is considered to be an invader. The results show that a random network is invaded not by strategy $Q$, but by strategy $H$ for $T>6$. On the other hand the SF-like network constantly shifts between $C$ and $H$ for $T>6$.

\section{ACKNOWLEDGMENTS}

Work by J. Sładkowski was supported by the Polish National Science Center under the project number UMO2011/01/B/ST6/07197, Ł. Pawela was supported by the Polish National Science Centre under the grant number N N519 442339.

Numerical simulations presented in this work were performed on the "Leming" and "Kapibara" computing systems of The Institute of Theoretical and Applied Informatics, Polish Academy of Sciences.
[1] M. Nowak and R. May, Nature 359, 826 (1992), URL http://www.ped.fas.harvard.edu/people/faculty/ publications_nowak/Nature92.pdf

[2] G. Szabó and G. Fáth, Physics Reports 446, 97 (2007), URL http://dx.doi.org/10.1016/j.physrep. 2007.04 .004

[3] E. W. Piotrowski and J. Sladkowski, arXiv:quantph/0211191 (2002), Int. J. Theor. Phys. 42 (2003) 1089, URL http://arxiv.org/abs/quant-ph/0211191

[4] J. Eisert, M. Wilkens, and M. Lewenstein, Physical Review Letters 83, 3077 (1999), URL http://link.aps. org/doi/10.1103/PhysRevLett.83.3077

[5] J. Miszczak, P. Gawron, and Z. Puchała, Quantum Information Processing pp. 1-13 (2011), URL http://www.springerlink.com/index/10.1007/ s11128-011-0322-2.

[6] Q. Li, A. Iqbal, M. Chen, and D. Abbott, Physica A 391, 3316 (2011), URL http://dx.doi.org/10.1016/j . physa.2012.01.048.

[7] J. Du, H. Li, X. Xu, X. Zhou, and R. Han, Physics Letters A 302, 229 (2002), URL http://dx.doi.org/10.1016/ S0375-9601(02)01144-1.

[8] J. Du, H. Li, X. Xu, M. Shi, J. Wu, X. Zhou, and R. Han, Physical Review Letters 88, 137902 (2002), URL http://link.aps.org/doi/10.1103/PhysRevLett.88. 137902 .

[9] P. Lou, Physica Status Solidi (b) 241, 1343 (2004), URL http://dx.doi.org/10.1002/pssb.200302009

[10] Z. Puchała, Quantum Information Processing pp. 1-8 (2012), URL http://dx.doi.org/10.1007/ s11128-012-0391-x.
[11] H. Guo, J. Zhang, and G. J. Koehler, Decision Support Systems 46, 318 (2008), URL http://dx.doi.org/10. 1016/j.dss.2008.07.001.

[12] E. W. Piotrowski and J. Sladkowski, Quantum Information Processing 1, 395 (2003), URL http://dx.doi.org/ 10.1142/S0219749903000279.

[13] B. Arfi, Theory and Decision 59, 127 (2005), URL http: //dx.doi.org/10.1007/s11238-005-8632-4

[14] J. Sladkowski, Physica A 324, 234 (2003), URL http: //dx.doi.org/10.1016/S0378-4371(02)01859-9.

[15] E. W. Piotrowski, J. Sladkowski, and J. Syska, Physica A 318, 516 (2003), URL http://dx.doi.org/10.1016/ S0378-4371(02)01370-5.

[16] F. Riccardo, Journal of Mathematical Psychology 53, 415 (2009), URL http://dx.doi.org/10.1016/j.jmp. 2009.02.002

[17] J. R. Busemeyer, Z. Wang, and J. T. Townsend, Journal of Mathematical Psychology 50, 220 (2006), URL http: //dx.doi.org/10.1016/j.jmp.2006.01.003

[18] E. W. Piotrowski and J. Sladkowski, Physica A 345, 185 (2005), URL http://dx.doi.org/10.1016/j . physa.2004.07.002.

[19] C. Berge, Graphs and hypergraphs, vol. 6 (Elsevier, 1976).

[20] A. Barabási and R. Albert, Science 286, 509 (1999), URL http://dx.doi.org/10.1126/science.286.5439.509.

[21] J. Chappell, A. Iqbal, and D. Abbott, PloS one 6, e21623 (2011), URL http://www.plosone.org/article/info\% 3Adoi\%2F10.1371\%2F journal. pone.0021623

[22] S. Benjamin and P. Hayden, Physical Review A 64, 030301 (2001), URL http://link.aps .org/doi/10. 1103/PhysRevA.64.030301.

[23] F. C. Santos, J. F. Rodrigues, and J. M. Pacheco, Physical Review E 72, 056128 (2005), URL http://link .aps. org/doi/10.1103/PhysRevE.72.056128. 